\title{
Modeling Dielectric Charged Drop Break Up using an Energy Conservation Method
}

\author{
J. S. Shrimpton \\ School of Engineering Sciences \\ Southampton University \\ Southampton, UK
}

\begin{abstract}
In the light of experimental evidence a previously published model to predict the charge and mass redistribution when charged dielectric drops break up has been updated. In particular we have taken the dielectric nature of the liquid, the existence of an external electric field and used photographic evidence of drop break up as a basis for geometrical assumptions that are physically realistic. We have assessed the sensitivity of the charge redistribution assumption and the improved model compares well with the recent accurate experimental evidence. The results apply to ratio of quantities of mass and charge, making the model extremely simple and economical to apply to multi-dimensional charged spray computer codes in order to predict evaporating charged sprays accurately.
\end{abstract}

Index Terms - Charge injection, liquid drop, dielectric, stability.

\section{INTRODUCTION}

MANY processes rely on evaporating multiphase flows and recently this has been extended to those that contain electric charge for coating processes [1]. Electrostatic atomization forms a number of fine, charged droplets [2] which disperse more readily than their uncharged counterparts [3], and may be directed by electric fields [4]. Such droplets are particularly well suited to coating applications such as painting, printing and crop spraying [5] as well more exotic applications such as combustion [6]. There is therefore a need to predict the outcome of the charge and mass redistribution from a parent drop to its products when the parent becomes critically unstable. As evaporation takes place, the radius of the droplet will reduce whilst the amount of charge carried remains constant [7]. As a result, the drop will inevitably reach a size where it is carrying the limiting amount of charge. Further evaporation implies that this limit is exceeded and the droplet will become unstable and break up. The stability limit is known as the Rayleigh Limit, in deference to Lord Rayleigh [8], who used a perturbation method to define the maximum charge a conducting drop, in vacuum, with no external disturbance present may hold. However, in a companion paper [9] we show that recent published experimental work proves that break up occurs before and not at the Rayleigh Stability Limit and we suggest an extension. Further, when break up does occur, then mass of the order $1 \%$ to $5 \%$ is ejected from the parent

Manuscript received on19 November 2007, in final form 10 June 2008. drop and the siblings created carrying a charge the order of $15 \%$ that of the parent. In addition to the quantitative experimental work on drop break up results listed in Shrimpton [9], Gomez and Tang [10] presented images of the disruption process. A reproducible disruption pattern is observed in where a large parent drop breaks up into a slightly smaller residual and a number of much smaller, near identical siblings, typically one eighth of the parent diameter. Given that the sibling droplets are of the order one eighth the diameter of the parent then this suggests, for a mass loss of about $3 \%$, that of the order of 15 sibling droplets should be produced. The deterministic and repeatable nature of the break up process irrespective of drop diameter lends itself the development of a break up model of the process. Many authors (e.g. [11]) have examined the dynamics of drop break up with much success, but to develop a sub-model for use in computational charged spray models [3] this approach is far too expensive in terms of computer resources. Roth and Kelly [12] proposed a model based simply upon mass, charge and energy conservation between initial and final energy states using the following assumptions:-

i. The starting drop was at rest and charged to the Rayleigh Limit.

ii. The effect of the external electric field was negligible.

iii. The break-up was isothermal, and viscous losses, further evaporation and aerodynamic effects were negligible.

iv.The siblings were all identical.

v. The siblings were emitted in geometrically regular patterns. 
We develop this model, only retaining assumptions (iii) and (iv) using the following energy conservation law,

$$
\left[W_{s}+W_{e}\right]_{0, t=0}=\left[W_{s}+W_{e}\right]_{1, t=\infty}+n\left[W_{s}+W_{e}+W_{k}\right]_{2, t=\infty}
$$

Equation (1) states that the pre-break up drop surface $\left(\mathrm{W}_{\mathrm{S}}\right)$ and electrostatic $\left(\mathrm{W}_{\mathrm{e}}\right)$ energy is equivalent to the post break up surface and electrostatic energies of a residual drop (subscript 1) and $\mathrm{n}$ siblings (subscript 2), together with the stored energy due to the proximity of the charged products at break up. We use the definitions of $\mathrm{W}_{\mathrm{S}}$ and $\mathrm{W}_{\mathrm{e}}$ as proposed by Roth and Kelly (equation (1), [12]), and they are not repeated here. In the light of more recent experimental evidence (reviewed in [9]), the assumption that the initial droplet would hold the Rayleigh Limit charge, used in the Roth and Kelly model, has been found to be inaccurate. In addition, the break-up geometry has since been observed and recorded, and not found to reflect the assumptions that were made in the formulation of the model. The assumption that an external electric field has no effect on the break up process may also be incorrect for dielectric drops, particularly so within charged spray plumes where effective space charge and electric field magnitudes may be large. Lastly some of the results are unusual, for instance the Roth and Kelly model only permitted solutions between 2 and 7 siblings and the sibling to parent mass ratios at between 0.14 and 0.20 which are much higher than recent, more accurate, experimental evidence suggests.

The work described in this paper therefore investigates how, (a), the initial charge of the parent $\mathrm{Q}_{0}$, (b), a more realistic break up geometry, (c), the $W_{k}$ definition and, (d), how the charge redistribution assumption affects the model behavior.

\section{BREAK UP MODEL: ORIGINAL FORMULATION}

Since the work of Roth and Kelly is central to this study, an analysis was performed to ensure that we could reproduce their results and thus understand the detailed behavior of this model approach. In outline, a solution to the energy balance, (1), is obtained by assuming a number of sibling droplets. The solution domain is then searched by varying the residual droplet size to find the value which gives the minimum error between the initial and final states. The order of magnitude of the terms involved in the calculations in SI units is small, for example $Q \sim 10^{-13} \mathrm{C}$ and $m \sim 10^{-12} \mathrm{~kg}$. In order to minimize rounding errors in the numerical model, the expressions given in this paper were nondimensionalized, e.g. $Q^{*}=Q / Q_{\text {ref }}$. By suitable choice of reference quantities, given in table 1 , the non-dimensional parameters are normalized to be of order one.
Table 1 . Reference quantities.

\begin{tabular}{|c|c|c|}
\hline Length & $r_{\text {ref }}$ & $r_{0}$ \\
\hline Surface Tension & $\gamma_{\text {ref }}$ & $\gamma$ \\
\hline Permittivity & $\varepsilon_{\text {ref }}$ & $\varepsilon_{0}$ \\
\hline Mass & $m_{r e f}$ & $m_{0}$ \\
\hline Charge & $Q_{\text {ref }}$ & $8 \pi \sqrt{r_{r e f}^{3} \varepsilon_{r e f} \gamma_{r e f}}$ \\
\hline Electric Field & $E_{r e f}$ & $\sqrt{\frac{\gamma_{r e f}}{r_{r e f} \varepsilon_{0}}}$ \\
\hline Energy & $W_{r e f}$ & $r_{r e f}{ }^{2} \gamma_{r e f}$ \\
\hline
\end{tabular}

Equations $(1,3,9,10)$ of Roth and Kelly [12] for initial charge $\left(\mathrm{Q}_{0}\right)$, electrostatic energy and surface energy $\left(\mathrm{W}_{\mathrm{e}}\right.$ and $\left.\mathrm{W}_{\mathrm{S}}\right)$, residual-sibling and sibling-sibling separation distances $\left(\mathrm{d}_{\mathrm{i}}\right.$ and $\left.\mathrm{d}_{\mathrm{ij}}\right)$, residual charge $\left(\mathrm{Q}_{1}\right)$, sibling charge $\left(\mathrm{Q}_{2}\right)$, interaction energy $\left(\mathrm{W}_{\mathrm{K}}\right)$, and residual mass were normalized to give the terms given below.

$$
\begin{aligned}
& Q_{0}{ }^{*}=Q_{\text {Ray }}{ }^{*}=\sqrt{r^{* 3} \varepsilon_{g}{ }^{*} \gamma^{*}} \\
& W_{S}^{*}+W_{e}^{*}=4 \pi r^{* 2} \gamma^{*}+8 \pi Q^{* 2} / \varepsilon_{g}{ }^{*} r^{*} \\
& d_{i j}^{*}, d_{i}^{*}=r_{2}{ }^{*} \sinh \left(x_{1}^{*} / r_{2}{ }^{*}\right)\left[\cosh \left(x_{1}{ }^{*} / r_{2}{ }^{*}\right)-1\right]+x_{1}{ }^{*} \\
& +r_{2}{ }^{*} \sinh \left(x_{2}{ }^{*} / r_{2}{ }^{*}\right)\left[\cosh \left(x_{2}{ }^{*} / r_{2}{ }^{*}\right)-1\right]+x_{2}{ }^{*} \\
& x_{k}^{*}=r_{2}{ }^{*} \cosh ^{-1}\left(1 / 2\left\{1+\sqrt{1+4 r_{k}^{*} / r_{2}^{*}}\right\}\right) \\
& Q_{1}^{*}=Q_{0}^{*}\left[1+n^{1 / 3}\left(\frac{r_{0}^{*}}{r_{1}^{*}}\right)^{2}\left\{1-\left(\frac{r_{1}^{*}}{r_{0}^{*}}\right)^{3}\right\}\right]^{-1 / 3} \\
& Q_{2}^{*}=\left(Q_{0}^{*}-Q_{1}^{*}\right) / n \\
& W_{k}^{*}=\frac{16 \pi n Q_{1}^{*} Q_{2}{ }^{*}}{\varepsilon^{*} d_{i}^{*}}+\frac{8 \pi}{\varepsilon^{*}} \sum \frac{Q_{2}{ }^{* 2}}{d_{i j}{ }^{*}} \\
& w_{k}{ }^{*}=8 \pi n^{2} Q_{2}{ }^{2} / \varepsilon^{*} d_{i}^{*} f(n) \\
& m_{1}^{*}=r_{1}^{* 3}
\end{aligned}
$$

Equation (7) gives the definition of interaction energy, where the last term represents the sibling-sibling interactions. Roth and Kelly modeled this sibling-sibling interaction using an approximation, as defined by equation (8), where $f(n)$ was a empirical function that was not defined. When this procedure was implemented, the results failed to match neither the magnitude nor the trends that were published by Roth and Kelly. 
An investigation identified a number of problems in the original work:

An error was identified in the expression for the charge on the residual droplet (equation (8) of [12]), the exponent of the denominator is $1 / 3$. We use a simpler expression, which does involve the sibling radius explicitly.

$$
Q_{1}^{*}=Q_{0}^{*} /\left(1+n\left(r_{2}^{*} / r_{1}^{*}\right)^{2}\right)
$$

Equation (10) is also less susceptible to round off error than the original, since the ratios in the original expression are very close to unity, and are then raised to various powers. Since an alternative non-dimensionalizing procedure has been used in our work, the more complicated expression is no longer required, although was retained for our initial comparative tests. The original form of the $f(n)$ function in the kinetic energy term (equation 11 of [12]) is of unknown form, but represents the ratio of the interaction energy for $\mathrm{n}$ siblings to that for the limiting case of spherical shell. Roth and Kelly [12] approximated $\mathrm{f}(\mathrm{n})$ by using a cubic polynomial between the values at $n=2,3,8$ and 30 , where 30 siblings represents the limiting case and $f(n)=1$. However, for $\mathrm{n}=8$ not all of the sibling-sibling interaction distances are identical, which introduces complications. The form of the function was found to have a significant effect on the results, so an expression for our calibration was developed for the kinetic energy without recourse to an approximation, i.e. dispensing with the $f(n)$ term entirely:

$$
W_{k}^{*}=\frac{16 \pi n Q_{2}^{*}}{\varepsilon^{*}}\left[\frac{Q_{1}^{*}}{d_{i}^{*}}+\left(\frac{n-1}{2}\right) \frac{Q_{2}{ }^{*}}{d_{i j}{ }^{*}}\right]
$$

This formulation implies that the procedure is restricted to numbers of siblings for which the sibling-sibling interaction distances (dij) are all equal and can be found analytically. These break-up geometries and the sibling-sibling interaction distances are given in Table 2 .

Table 2. Analytical break-up geometry for $\mathrm{f}(\mathrm{n})$ verification.

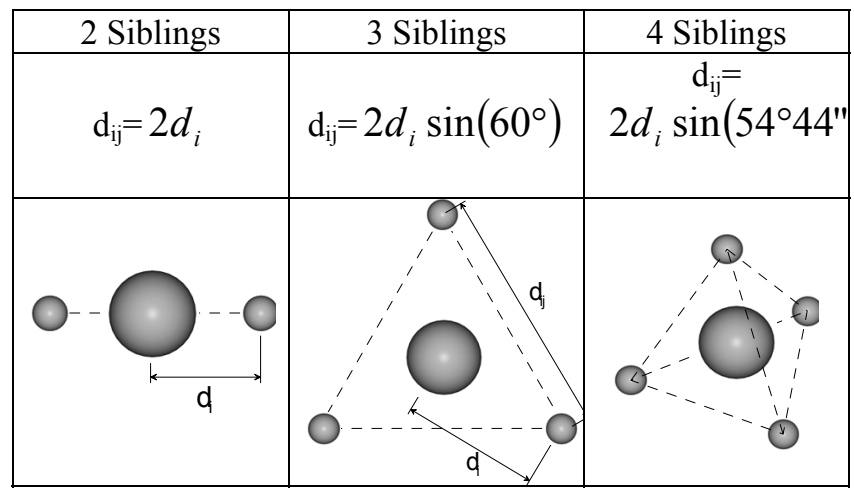

Although the number of cases that can be compared is limited, it is still sufficient to calibrate our work with that of Roth and Kelly whilst avoiding addressing the accuracy of the $f(n)$ term. The procedure was found to yield a very good match with the published results, see table 3 .
Table 3. Comparison between [12] and the present work.

\begin{tabular}{|c|c|c|c|c|c|c|c|c|c|c|}
\hline & R\&K & New & $\begin{array}{c}\text { Error } \\
\%\end{array}$ & R\&K & New & $\begin{array}{c}\text { Error } \\
\%\end{array}$ & R\&K & New & $\begin{array}{c}\text { Error } \\
\%\end{array}$ \\
\hline $\mathrm{N}$ & \multicolumn{3}{|c|}{ Mass Ratio } & \multicolumn{3}{|c|}{ Charge Ratio } & \multicolumn{3}{|c|}{$\begin{array}{c}\text { Formation } \\
\text { Distance }\end{array}$} \\
\hline 2 & 0.994 & 0.994 & $\mathbf{0 . 0 4 3}$ & 0.960 & 0.962 & $\mathbf{0 . 1 8 6}$ & 1.48 & 1.47 & $\mathbf{- 0 . 9 1 6}$ \\
\hline 3 & 0.990 & 0.990 & $\mathbf{- 0 . 0 2 0}$ & 0.937 & 0.936 & $\mathbf{- 0 . 0 9 7}$ & 1.49 & 1.49 & $\mathbf{0 . 2 1 9}$ \\
\hline 4 & 0.986 & 0.986 & $\mathbf{0 . 0 3 3}$ & 0.913 & 0.916 & $\mathbf{0 . 3 3 6}$ & 1.51 & 1.49 & $\mathbf{- 1 . 1 3 0}$ \\
\hline & \multicolumn{3}{|c|}{ Sibling Charge } & \multicolumn{3}{|c|}{ Sibling Size } & \multicolumn{3}{|c|}{$\begin{array}{c}\text { Electrostatic } \\
\text { Energy }\end{array}$} \\
\hline 2 & 0.134 & 0.362 & $\mathbf{1 7 0 . 2}$ & 0.144 & 0.141 & $\mathbf{- 2 . 2 9 8}$ & 0.981 & 0.967 & $\mathbf{- 1 . 4 7 0}$ \\
\hline 3 & 0.133 & 0.365 & $\mathbf{1 7 4 . 7}$ & 0.149 & 0.150 & $\mathbf{0 . 9 0 3}$ & 0.970 & 0.946 & $\mathbf{- 2 . 4 8 7}$ \\
\hline 4 & 0.131 & 0.359 & $\mathbf{1 7 3 . 9}$ & 0.154 & 0.151 & $\mathbf{- 2 . 1 7 3}$ & 0.958 & 0.930 & $\mathbf{- 2 . 8 8 4}$ \\
\hline
\end{tabular}

The only significant variation concerned the charge held on the sibling droplets. This is assumed to lie either in an error in the published work [12], or in the definition of 'sibling charge' which is not given explicitly in the paper of Roth and Kelly. The latter hypothesis seems more likely as we are able to reproduce the electrostatic energy results to within a few percent. As a whole, these results give confidence both in the present procedure and in our interpretation of the work by Roth and Kelly and we now use this result as the basis for an improved model.

\section{BREAK UP MODEL : NEW FORMULATION}

We extend the model of Roth and Kelly in three ways. Firstly we introduce a realistic break up geometry, secondly we allow for non-Rayleigh Limit charge to induce break up and thirdly we examine the effect of differing charge redistribution assumptions on the predictions. By comparison with the recent experimental results (Figures 5, 6 and 8 of [10]), it is observed that the emission of sibling droplets from many sites on the surface of the parent drop as assumed by Roth and Kelly is not realistic. As a consequence, two different geometries are proposed, both based on the observed linear break-up pattern as shown in Figure 1 where separation of the droplets is again based on the formation of a catenary surface between two droplets (see equation (10) of Roth and Kelly [12]).

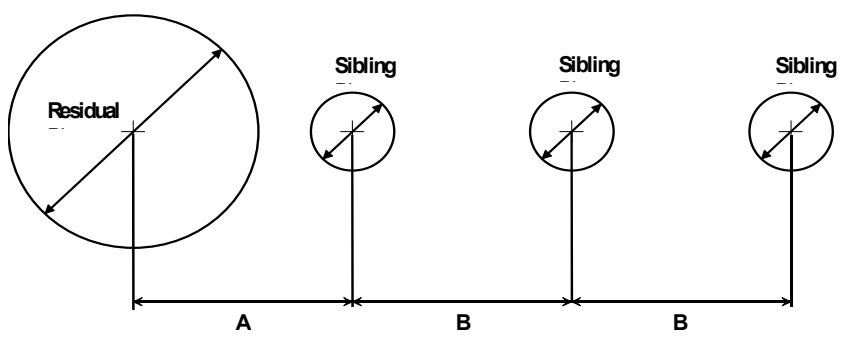

Figure 1. Residual-sibling separation.

The 'wide spacing' linear break-up geometry assumes that all the droplets are equally spaced, and the separation is equal to the initial drop separation distance, ie in Figure $1 \mathrm{~A}=\mathrm{B}=\mathrm{d}_{\mathrm{i}}$ 
where $d_{i}$ is calculated from equations (3) and (4) using $r_{1}$ and $\mathrm{r}_{2}$. This would be the result if each sibling droplet were emitted individually in turn from a single location on the residual surface and the sibling droplet velocities did not vary. In the 'close spacing' geometry the residual-sibling and the sibling-sibling separation distance are different, which would be the consequence of the break-up of a jet that forms from the parent droplet, and then collapses to form a number of droplets simultaneously. Here A in Figure 1 is calculated as above and $B=d_{i j}$ where $d_{i j}$ uses $r_{2}$ twice in equations (3) and (4). As shown by Table 4 a quantitative analysis of Figure $6 \mathrm{~b}$ of Gomez and Tang [10], from scanning the figure, shows that the 'close' geometry is seen to provide a close match to the measured values.

Table 4. Comparison of measured and calculated geometry, using catenary assumption (arbitrary units).

\begin{tabular}{|l|c|}
\hline Break-up Radius Sibling-Sibling Distance \\
\hline Minor Diameteryarent) \\
\hline Diagram reproduced exactly from Gomez and Tang $[10]$ \\
\hline
\end{tabular}

Using the 'wide spacing' geometry, the calculated siblingsibling distance would equal to the calculated break-up radius. In this case, this would calculate the sibling-sibling distance as 41 units, compared to 18 calculated using the 'close' assumption and the actual measured value being 23 units.

Regardless of the actual separations, we still need to calculate the stored kinetic energy of the post break up geometries. For the case when every droplet is assumed to interact with every other droplet, this leads to equations (12) and (13), which it noted, do not now rely on any approximations of $\mathrm{f}(\mathrm{n})$ in terms of sibling number.

$W_{k}=\sum_{k=1}^{n} \frac{Q_{1} Q_{2}}{4 \pi \varepsilon k d_{i}}+\sum_{k=1}^{n-1} \frac{n-k}{k} \frac{Q_{2}^{2}}{4 \pi \varepsilon d_{i}}$

$$
W_{k}=\sum_{k=1}^{n} \frac{Q_{1} Q_{2}}{4 \pi \varepsilon k d_{i}}+\sum_{k=1}^{n-1} \frac{n-k}{k} \frac{Q_{2}^{2}}{4 \pi \varepsilon d_{i j}}
$$

Equation (12) assumes equal sibling-sibling and residualsibling spacing, and equation (13) supposes sibling-sibling spacing is closer than the residual-sibling spacing.

In addition, for the case where the siblings were assumed to interact only with their nearest neighbor, and with the residual:

$$
\begin{aligned}
& W_{k}=\sum_{k=1}^{n} \frac{Q_{1} Q_{2}}{4 \pi \varepsilon k d_{i}}+\frac{(n-1) Q_{2}^{2}}{4 \pi \varepsilon d_{i}} \\
& W_{k}=\sum_{k=1}^{n} \frac{Q_{1} Q_{2}}{4 \pi \varepsilon k d_{i}}+\frac{(n-1) Q_{2}^{2}}{4 \pi \varepsilon d_{i j}}
\end{aligned}
$$

Similarly, equation (14) assumes equal sibling-sibling and residual-sibling spacing, and equation (15) supposes siblingsibling spacing is closer than the residual-sibling spacing.

We know that dielectric drops break up at sub-Rayleigh Limit charges and we have developed a new expression [9] that correlates well with the break up of charged drops in charges spray plumes [10] and this is used as the charge on the parent at break-up,

$$
\frac{Q_{f}}{Q_{\text {Ray }}}=\left(\frac{\varepsilon_{g}}{2 \varepsilon_{g}-1}\right)\left\{1-\frac{3}{2} E_{0} \sqrt{\frac{r \varepsilon_{0}}{\varepsilon_{g} \gamma}}\left(\frac{\varepsilon_{d}-\varepsilon_{g}}{\varepsilon_{d}+2 \varepsilon_{g}}\right)\right\}
$$

Two different assumptions were proposed for investigation of post-fission charge distribution between the residual and sibling droplets. Firstly, the assumption stated by Roth and Kelly to give most realistic results in their model is that the same surface charge density exists on the residual and sibling droplets. This assumption leads to the expression:

$Q_{1}=Q_{0} /\left(1+n\left(r_{2} / r_{1}\right)^{2}\right)$

An alternative assumption states that the surface charge density on the sibling droplets after fission equals that on the parent droplet before fission, with the remainder being carried on the residual. This leads to:

$Q_{2}=\left(r_{2} / r_{0}\right)^{2} Q_{0}$

We now discuss the methodology of the model and present results to define its behavior.

\section{DISCRETIZATION PROCEDURE AND DEFINITION OF BREAK UP CONDITION}

As in the model developed to reproduce the results of Roth and Kelly, for an assumed number of siblings and break up geometry the model searches through a defined range of residual sizes and at each applies the specified kinetic energy and charge redistribution assumption. Provided all the charge is of the same sign, then the energy balance is performed and 
the residual droplet size that corresponds to the minimum error in the energy balance is presented as the solution. There are a number of validation tests built in to the model and most importantly, the model not only finds the minimum error, but only accepts a result if the error has changed sign for a particular number of siblings during the analysis. We also ensure that our most exact energy balance is not simply one of the first few steps of the search algorithm, where sibling drops can be exceedingly small and the initial and final energies are similar regardless of boundary conditions. We also investigated the sensitivity of the results to the number of residual sizes investigated and found that providing we evaluate at least $10^{3}$ sizes the results are independent of step size.

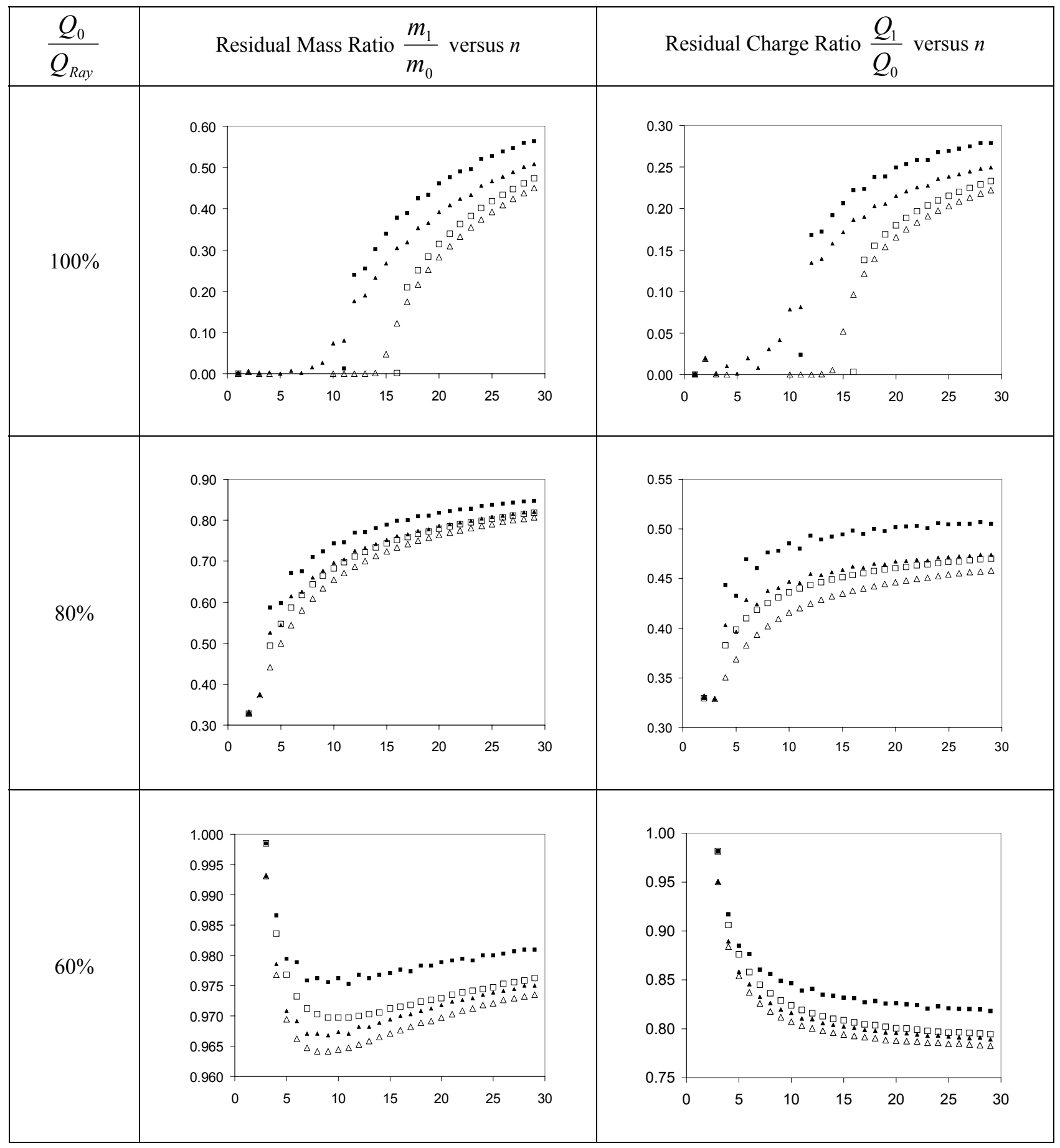

Figure 2. Residual Mass and Charge Ratio assuming $Q_{1}=Q_{0} /\left(1+n\left(r_{2} / r_{1}\right)^{2}\right)$, eqn (17), for the linear geometries defined by equations (12), $\Delta$

(14), $\triangle$ and (15) $\square$. 


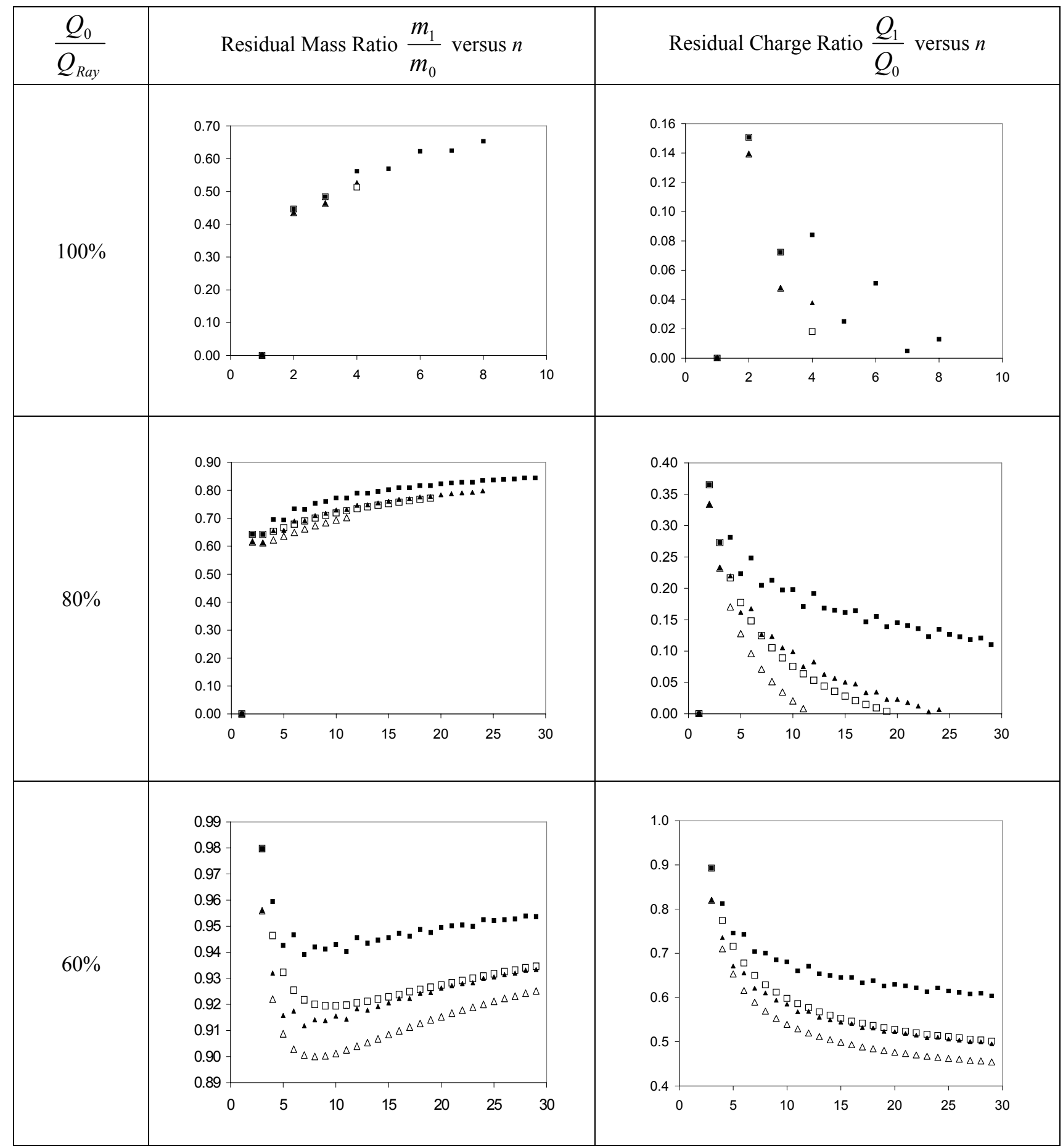

Figure 3. Residual mass and charge ratio assuming $Q_{2}=\left(r_{2} / r_{0}\right)^{2} Q_{0}$, equation (18), for the linear geometries defined by equations (12),

(14), $\triangle$ and (15) $\square$.

\section{MODEL PREDICTIONS}

Calculations were carried out with the charge at break-up being $100 \%, 80 \%$ and $60 \%$ of the Rayleigh Limit [9]. This was to reflect the range of values observed in the experiments, and for this study reflects an increasing applied electric field, starting from zero field at $100 \%$ of the Rayleigh Limit. This approach allows the two halves of the analysis, prediction of critical charge and prediction of fission products to be decoupled and the effects considered separately. Figures 2 and 3 contain graphs showing the solutions for the residual droplet mass and charge, against number of siblings. Each Figure shows the results for a different charge redistribution 
assumption, and contains the data for all investigated initial conditions and kinetic energy assumptions. Both the trends and magnitudes of the results are similar regardless of the assumptions used in the kinetic energy and break up geometry assumptions. This is reasonable, because the range of assumed break up geometries was quite restricted, in order to reflect reality, and the sibling size (and hence energy) are small relative to the residual drop. The rank of the results is always the same, however, with the model containing closer siblings predicting the larger residual size. If all the droplets are assumed to interact with each other, then the residual is larger still. The assumption regarding the redistribution of charge during the disruption however has an important effect on the predicted results. The effect on the mass of the residual is not great, but the charge on the residual can vary by a factor of four, with all other parameters held constant. For example with an initial charge of $60 \%$ of the Rayleigh Limit and 30 sibling droplets produced, the charge on the residual can vary from $20 \%$ to $80 \%$ of the parent charge, depending on the charge redistribution method assumed. The most important parameter is the specification of the initial charge of the drop at break-up. This has a very significant effect on the outcome of the model, and was not investigated by Roth and Kelly, since then the accuracy and validity of the Rayleigh Limit was assumed. Also the charge on the sibling droplets never exceeds the Rayleigh Limit for the droplet. This suggests a degree of robustness in the model, since no explicit check for this limit was enforced, but arises as a result of the other assumptions. Likewise the combinations of assumptions in the model, all based on experimental data predict mathematically valid solutions over a wide range of possible sibling numbers.

The key results from the most reliable experimental evidence was that the residual is of the order of $95 \%$ or more of the mass of the parent, and holds of the order $75 \%$ or more of the charge of the parent drop. If the numerical results are compared to the experimental evidence, then an inspection of Figures 2 and 3 reveal that most combinations of initial conditions and charge redistribution do not predict useful results. However, for a parent droplet charged to $60 \%$ of the Rayleigh Limit at break up, when the surface charge of sibling and residual are equal is the exception and very closely matches the more reliable experimental evidence, for numbers of siblings greater than five. Further it can be shown that the sibling droplet charge is between $15 \%$ and $23 \%$ of the Rayleigh Limit which matches very well with experimental data.

\section{CONCLUSION}

A previously documented charged drop break up model has been re-examined and improved by considering a more realistic break up geometry, a range of charge redistribution assumptions and break up at sub-Rayleigh Limit drop charges. All these modifications have been based upon established experimental work which became available since the development of the original model in 1983. The model is appealing because the results are correlated in the form of ratios, which means that the model applies equally to all drops. This would lead to a very computationally efficient method of charged drop break up when predicting the development of a transient polydisperse charged spray under evaporating conditions. The most accurate model results occur under the following conditions: when the initial drop charge is near $60 \%$ of the Rayleigh Limit, the break up geometry is as shown in Figure 1, but where the sibling drops are closely spaced and are all considered in the kinetic energy calculation, and lastly the charge density on the residual drops and the siblings are equal. Using these assumptions, $\mathrm{Q}_{0} / \mathrm{Q}_{\text {ray }}=0.60$, $\mathrm{m}_{1} / \mathrm{m}_{0}=0.975-0.98$ and $\mathrm{Q}_{1} / \mathrm{Q}_{0}=0.82-0.88$ and $\mathrm{n}=5-25$, compared with experimental data $[10]$ of $\mathrm{Q}_{0} / \mathrm{Q}_{\mathrm{ray}}=0.70-0.80$, $\mathrm{m}_{1} / \mathrm{m}_{0}=0.97$ and $\mathrm{Q}_{1} / \mathrm{Q}_{0}=0.85$ and $\mathrm{n}=\sim 15$.

\section{REFERENCES}

[1] C.H. Chen, M.H.J. Edmond, E.M. Kelder, B. Meester and J. Schoonman, "Electrostatic sol-spray deposition of nano-structured ceramic thin films", J. Aerosol Sci., Vol. 30, pp. 959-967, 1999.

[2] A.M. Ganan-Calvo, "The surface charge in electrospraying: Its nature and its universal scaling laws", J. Aerosol Sci., Vol 30, pp. 863-872, 1999.

[3] J.S. Shrimpton and Y. Laoonual, "Dynamics of Electrically-Charged transient evapourating sprays", Intern. J. Numerical Methods Eng., Vol. 67, pp.1063-1081, 2006.

[4] J. Domnick, T. Scheibe, G. Steigleder, G. Weckerle and Q. Ye, "Simulation of the Electrostatic Spray Painting Process with High Speed Rotary Bell Atomizers", Proc. Intern. Conf. Liquid Atomization and Spray Systems 2000, Paper S.617-624, Pasedana, California, USA, 2000.

[5] A.G. Bailey, "Theory and practice of electrostatic spraying", Atomization and Spray Tech., Vol. 2, pp. 95-134, 1986.

[6] J.S. Shrimpton and A.J. Yule, "Atomization, Combustion and Control of Charged Hydrocarbon Sprays", Atomization and Sprays, Vol. 11, pp. 1$32,2001$.

[7] S.E. Law, "Charge and mass flux in the radial electric field of an evaporating charged water droplet: An Experimental Analysis", IEEE Trans. Industrial Applications, Vol. 25, pp. 1081-1087, 1989.

[8] Lord Rayleigh, "On the Equilibrium of Liquid Conducting Masses Charged with Electricity", Phil. Mag., Series 5, Vol. 14, pp. 184-186, 1882.

[9] J.S. Shrimpton, "Dielectric Charged drop Break up at Sub-Rayleigh Conditions", IEEE Trans. Dielectr. Electr.Insul., Vol. 12, pp. 573-578, 2005.

[10] A. Gomez and K. Tang, "Charge and fission of droplets in electrostatic sprays", Phys. Fluids, Vol. 6, pp. 404-414, 1994.

[11] P.M. Adornato and R.A. Brown, "Shape and stability of electrostatically levitated drops", Proc. Roy. Soc. Lond. A, Vol. 389, pp. 101-117, 1983.

[12] D.G. Roth and A.J. Kelly, "Analysis of the disruption of evaporating charged droplets", IEEE Trans. Industrial Applications, Vol. 1A-19, pp. $771-775,1983$

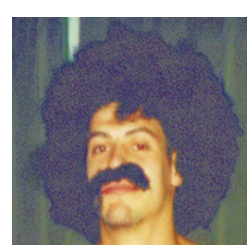

J. S. Shrimpton was born in Essex, UK in 1969. He received the B.Eng. degree from the University of Birmingham, UK in 1991, and the Ph.D. degree from the University of Manchester, UK in 1995. From 1998 to 2007 he was a Lecturer and EPSRC advanced research fellow at Imperial College London, and from December 2007 a Senior Lecturer at the University of Southampton, UK. 